\title{
DEPENDÊNCIA E SÍNDROME DE ABSTINÊNCIA DOS OPIOIDES: UMA REVISÃO NARRATIVA PARA IDENTIFICAR OS RISCOS RELACIONADOS AO USO INDEVIDO E/OU PROLONGADO DESSA CLASSE
}

\author{
OPIOID DEPENDENCE AND WITHDRAWAL SYNDROME: A NARRATIVE \\ REVIEW TO IDENTIFY RISKS RELATED TO THE MISUSE AND/OR \\ PROLONGED USE OF THIS CLASS
}

\author{
Juliane dos Santos Oliveira ${ }^{1}$ \\ Silvana Teixeira Corrêa ${ }^{2}$ \\ Aline Preve da Silva ${ }^{3}$ \\ Rafaela dal Piva ${ }^{4}$
}

RESUMO: Introdução: Os medicamentos opioides são indicados para o tratamento, controle e alívio de dores consideradas agudas e crônicas, por possuírem alto poder analgésico e hipnótico. Porém, o uso deste tipo de medicamento, principalmente quando indevido ou prolongado, pode oferecer diversos tipos de riscos à saúde do usuário, tais como o vício e a dependência, fatores que tem sido motivo de preocupação no Brasil e no Mundo. Objetivo: Apresentar evidências teóricas sobre a dependência e a síndrome de abstinência como riscos potenciais relacionados ao uso indevido e/ou prolongado de medicamentos opioides por usuários crônicos deste princípio ativo. Materiais e Métodos: No presente trabalho foi realizada uma revisão bibliográfica utilizando de estudos científicos publicados nos últimos is anos relacionados ao tema proposto. Resultados: a partir da análise dos materiais selecionados para a pesquisa, foram encontradas evidências teóricas de que a dependência e a síndrome de abstinência figuram como riscos potenciais relacionados ao uso indevido e/ou prolongado de medicamentos opioides por usuários crônicos deste princípio ativo. Conclusão: Entre os riscos oferecidos pelo uso indevido de opioides, as evidências teóricas apontam a dependência e a síndrome de abstinência, entre outros, tais como a tolerância, o vício, a overdose e até mesmo o óbito. Desta forma, cabe a reflexão da necessidade de mais pesquisas sobre o fenômeno da dor e sobre o uso dos opioides bem como o reforço constante na educação de quem os prescreve, garantindo a segurança do tratamento proposto.

Palavras-chave: Analgésicos. Dependência. Transtornos relacionados ao uso de opioides.

\footnotetext{
' Graduanda do curso de Farmácia CESUFOZ (Centro de Ensino Superior de Foz do Iguaçu).

${ }^{2}$ Graduanda do curso de Farmácia CESUFOZ (Centro de Ensino Superior de Foz do Iguaçu).

3 Docente do curso de Farmácia CESUFOZ (Centro de Ensino Superior de Foz do Iguaçu).

${ }_{4}^{4}$ Docente do curso de Farmácia CESUFOZ (Centro de Ensino Superior de Foz do Iguaçu).
} 
ABSTRACT: Introduction: Opioid drugs are indicated for the treatment, control and relief of pain considered acute and chronic because they have high analgesic and hypnotic power. However, the use of this type of medication, especially when improper or prolonged, can offer different types of risks to the user's health, such as addiction and dependence, factors that have been a matter of concern in Brazil and worldwide. Objective: To present theoretical evidence on dependence and withdrawal syndrome as potential risks related to the misuse and/or prolonged use of opioid drugs by chronic users of this active ingredient. Materials and Methods: a literature review of the narrative type was carried out through scientific materials published in the last I5 years related to the proposed theme. Results: from the analysis of the materials selected for the research, theoretical evidence was found that dependence and withdrawal syndrome are potential risks related to the misuse and/or prolonged use of opioid drugs by chronic users of this active ingredient. Conclusion: Among the risks offered by the misuse of opioids, theoretical evidence points to dependence and the withdrawal syndrome, among others, such as tolerance, addiction, overdose and even death. There is currently a great concern regarding the use of opioids in view of. Thus, it is worth reflecting on the need for more research on the phenomenon of pain and on the use of opioids, as well as constant reinforcement in the education of those who prescribe them, ensuring the safety of the proposed treatment.

Keywords: Opioid analgesics. Opioid addiction. Disorders related to the use of opioids. Opioid epidemic.

\section{INTRODUÇÃO}

Os opioides são utilizados individualmente ou associados a outros medicamentos. O tratamento é especialmente indicado no alívio das dores moderadas e severas. $\mathrm{O}$ alto poder analgésico e hipnótico garante o alívio das dores nos casos mais variados de enfermidades agudas. Porém, o uso deste tipo de medicamento, principalmente quando indevido ou prolongado, pode oferecer diversos tipos de riscos à saúde do usuário, tais como o vício e a dependência, fatores que tem sido motivo de preocupação no Brasil e no Mundo (CAMPOS et al., 2020; MELO et al., 2020).

De acordo com Calônego (2020, p. I8), a concentração no consumo de medicamentos opioides no mundo encontra-se sobretudo nos países da América do Norte, Europa Ocidental e Oceania, responsáveis por absorver "92\% da morfina disponível em todo o planeta". Isso se deve ao fato de que, nestas localidades, há um desequilíbrio na prescrição destes medicamentos acarretando desvio, mau uso e abuso 
destas substâncias e os consequentes riscos inerentes a esta prática, tais como o vício, a dependência e a overdose.

Especificamente no Brasil, o uso dos medicamentos opioides obedece a diversas normativas das autoridades competentes, exigindo-se prescrição médica adequada ao tratamento indicado. Apesar do país não figurar no rol mundial de maiores consumidores de medicamentos opioides, segundo Calônego (2020) e Leal e Alencar (2020), o Brasil é o maior consumidor de analgésicos opioides da América do Sul, porém este consumo é considerado bastante inferior àquele praticado nos países mais desenvolvidos.

Uma pesquisa publicada pela Fundação Oswaldo Cruz (FIOCRUZ) em 2018 revelou que no Brasil, entre os anos de 2009 e 2015, o número de vendas de opiáceos com prescrição médica cresceu em $465 \%$, sem contar as vendas clandestinas e ilegais destas substâncias no famoso mercado negro (PORTELA, 2018).

Alguns estudos relacionados aos riscos inerentes ao uso deste princípio ativo, tais como os estudos conduzidos por Melo et al. (2020), Campos et al. 2020 e Leal e Alencar (2020), indicam que a dependência e a síndrome de abstinência se configuram como riscos potenciais neste contexto e revelam a necessidade de mais pesquisas sobre o assunto.

Sendo assim, a presente pesquisa teve por objetivo apresentar evidências teóricas sobre a dependência e a síndrome de abstinência como riscos potenciais relacionados ao uso indevido e/ou prolongado de medicamentos opioides por usuários crônicos deste princípio ativo através de revisão de literatura do tipo narrativa.

\section{MATERIAIS E MÉTODOS}

A pesquisa foi realizada através de uma revisão bibliográfica do tipo narrativa através de materiais científicos publicados nos últimos is anos relacionados ao tema proposto.

O levantamento do material para o presente estudo teve como base os descritores ou palavras-chave "analgésicos opioides", “dependência de opioides", "transtornos relacionados ao uso de opioides" e "epidemia de opioides", todos disponíveis no site do DeCS (Descritores em Ciência da Saúde). 
A busca ocorreu na base de dados SciELO (Scientific Electronic Library Online) e no recuperador de documentos científicos Google Acadêmico, bem como em sites de universidades brasileiras federais e particulares, nas línguas inglesa, portuguesa e espanhola.

Como critérios de inclusão, optou-se pelos materiais publicados nos últimos is anos e que tinham relação com o tema proposto e com os objetivos da pesquisa, priorizando os artigos científicos disponíveis nas fontes citadas no item anterior. Foram excluídos os materiais que não se encaixam nos critérios de inclusão adotados.

Para identificar as publicações utilizadas para a pesquisa em questão, atentouse primeiramente para o título e leitura dos resumos dos materiais levantados a fim de verificar se estavam relacionados ao tema e objetivos propostos. Após esta etapa, foram selecionados os artigos científicos para leitura e análise na íntegra.

Uma vez selecionados os artigos relacionados ao tema, partiu-se para a identificação das evidências teóricas sobre a dependência e a síndrome de abstinência como riscos potenciais relacionados ao uso indevido e/ou prolongado de medicamentos opioides por usuários crônicos deste princípio ativo. Para tanto, os

materiais foram divididos de acordo com seu conteúdo na seguinte categorização: conceitos de opioides, orientações para o tratamento com opioides, conceitos de dores crônicas, riscos relacionados ao uso prolongado e/ou indevido de opioides, conceitos de dependência e síndrome de abstinência.

\section{REVISÃO BIBLIOGRÁFICA}

Os opioides, como o próprio nome denota, são substâncias derivadas do ópio. Este, por sua vez, é extraído da planta Papaver somniferum, pertencente à família das Papaveráceas, conhecida popularmente pelo nome de papoula (CAMPOS et al., 2020; MELO et al., 2020).

No Protocolo Clínico e Diretrizes Terapêuticas da Dor Crônica, aprovado pela Portaria № 1.083/2012 da Secretaria de Atenção à Saúde (SAS/MS, 2012) e do Ministério da Saúde (MS), opioides podem ser entendidos como fármacos analgésicos com alta potência e eficácia no tratamento a longo prazo de indivíduos acometidos por dores oncológicas, mistas ou neuropáticas, pois são mais eficientes que as classes: 
antidepressivos tricíclicos e a ANES, sendo esta sigla utilizada para se referir aos antiinflamatórios não esteroidais. O protocolo oferece, ainda, como exemplos de opioides fracos, tais como a codeína e o tramadol, e de opioides fortes, a morfina, a oxicodona, a metadona, a fentanila e a hidromorfona.

Os medicamentos opioides são alternativas para o tratamento de dores agudas e crônicas por possuírem potente eficácia terapêutica de efeito analgésico e hipnótico. São geralmente indicados para pacientes oncológicos, vítimas de queimaduras severas, tratamento de dores pós-cirúrgicas, indivíduos politraumatizados, entre outros (SOARES et al., 2008; ANTUNES, 2018; O'MALLEY; O'MALLEY, 2020).

Por serem os medicamentos alvos da presente pesquisa indicados no tratamento de dores agudas e crônicas, considera-se importante também definir a dor, de forma breve, à luz de referencial teórico.

Segundo a Associação Internacional para o Estudo da Dor (IASP), a dor é compreendida como "uma experiência sensitiva e emocional desagradável associada, ou semelhante àquela associada, a uma lesão tecidual real ou potencial" (SOUZA; BARROS, 2020, p. 294).

Em relação aos parâmetros e a distinção entre as dores aguda e crônica, de acordo com o Protocolo Clínico e Diretrizes Terapêuticas da Dor Crônica, aprovado pela Portaria SAS/MS № 1.083/2012, a dor considerada aguda é aquela cuja duração é inferior a 30 dias. A é dor classificada como crônica apresenta duração maior do que 30 dias e pode ser classificada da seguinte maneira: dor de predomínio nociceptivo, que está relacionada à lesão de tecidos ósseos, musculares ou ligamentares; dor de predomínio neuropático, compreendida como dor iniciada por lesão ou disfunção do sistema nervoso; e a dor mista, sendo esta última a mais comum e presente na prática clínica, e cujo próprio nome denota, envolve a presença das dores de predomínio neuropático e nociceptivo (SAS/MS, 2012).

No Brasil, segundo o Protocolo Clínico e Diretrizes Terapêuticas da Dor Crônica vigente, o tratamento da dor obedece às diretrizes propostas pela Organização Mundial de Saúde (OMS) através da chamada "Escada Analgésica”, publicada no ano de 1986 e composta de três degraus de orientação para este tipo de tratamento conforme 
intensidade da dor apresentada pelo paciente (SAS/MS, 2012; BICCA et al., 2012; CFF, 2016).

O primeiro degrau é indicado para o tratamento das dores consideradas leves através da recomendação do uso de medicamentos analgésicos simples e antiinflamatórios verificando-se, conforme o caso do paciente, a necessidade de incluir no tratamento também os fármacos adjuvantes, como antidepressivos, anticonvulsivantes ou relaxantes musculares. Já no segundo degrau, cujo tratamento é indicado em casos de dores de intensidade moderada e quando o primeiro degrau não surtiu efeito, adiciona-se o emprego de opioides considerados fracos e que podem ser associados aos analgésicos simples e anti-inflamatórios. O terceiro degrau, por sua vez, é recomendado para o tratamento de dores consideradas graves, de intensidade forte, cujo controle não foi possível através da aplicação dos medicamentos constantes no segundo degrau, ou seja, orienta-se a substituição do opioide considerado fraco para outro mais forte, associados ou não aos analgésicos simples ou anti-inflamatórios que, no Brasil, inclui fármacos como a morfina, a metadona e a oxicodona (SAS/MS, 20I2; BICCA et al., 2012; CALÔNEGO, 2020).

$\mathrm{Na}$ mesma linha de pensamento, Leal e Alencar (2020) alertam que para definir a dose ideal do princípio ativo a ser usado em determinado tratamento, é indicado o método da titulação, ou seja, administrar a dose mínima recomendada e ir aumentado a mesma de forma gradual até atingir o efeito analgésico desejado, buscando manter os efeitos adversos toleráveis.

Em consonância, Kraychete, Garcia e Siqueira (2014) salientam a importância do acompanhamento e do monitoramento do emprego dos opioides no tratamento da dor, principalmente a longo prazo, buscando uma avaliação constante do paciente e de sua evolução, alinhando questões relacionadas a risco-benefício, objetivos e indicações do tratamento.

Em matéria publicada pelo Conselho Federal de Farmácia sobre os riscos inerentes ao uso prolongado de opioides, é necessário estabelecer um meio termo, um equilíbrio no emprego dos opioides em tratamentos que demandam planejamento a acompanhamento adequados (CFF, 2016). 
É relevante destacar que no Brasil existem inúmeras normativas com a finalidade de controlar e regulamentar a venda, a prescrição e a utilização de medicamentos que exigem um cuidado mais aprofundado devido aos riscos inerentes ao seu emprego em tratamentos de diversas naturezas. De acordo com Campos et al. (2020) e Calônego (2020), merecem destaque a Portaria SVS/MS № 344/1998 e a Portaria SAS/MS № 1.083/2012. A primeira traz em seu teor, entre outras, diretrizes importantes relacionadas à documentação e formulários necessários na dispensação dos medicamentos opioides, sendo estes sujeitos ao controle especial. Já a segunda oferece o Protocolo Clínico e Diretrizes Terapêuticas da Dor Crônica é bastante completo, disponibilizando conceitos, definições e orientações importantes em relação ao uso de medicamentos opioides no tratamento da dor crônica, tais como critérios diagnósticos, critérios de inclusão e exclusão e diretrizes de tratamento, controle e avaliação.

Toda a regulamentação existente no Brasil em relação aos medicamentos opioides, entre outros, baseia-se na preocupação em relação aos riscos que o seu uso oferece. Portanto, além de estabelecer formas de controle, as normativas buscam orientar os profissionais da saúde, principalmente médicos e farmacêuticos, no que concerne à correta dispensação e prescrição destes medicamentos.

Especificamente sobre o farmacêutico, Campos et al. (2020) apontam que este profissional tem por objetivo orientar o usuário para que esta classe de medicamentos seja administrada de forma segura. De acordo com os autores, o farmacêutico deve se atentar mais às atividades clínicas, que envolvem as orientações em relação ao medicamento prescrito, tais como a maneira correta do seu uso no que diz respeito às dosagens e intervalos entre as tomadas e a relevância de seguir a prescrição com exatidão para o alcance de resultados positivos; também tem a função de alertar sobre os possíveis riscos e efeitos adversos, bem como avaliar a prescrição analgésica e, diante da percepção de incompatibilidade nesta última, comunicar o fato ao usuário.

Em relação aos riscos relacionados ao uso de medicamentos opioides, principalmente ao seu consumo indevido e/ou prolongado, a partir da análise dos dados coletados nas fontes pesquisadas para o presente estudo, foi possível elencar que, entre os riscos potenciais advindos desta prática, encontram-se a intoxicação, a 
tolerância, a dependência, o vício, a síndrome de abstinência, a overdose e o óbito (SWIFT; LEWIS, 2010; MELO et al., 2020; OMS, 2020).

A preocupação com os riscos relacionados ao uso dos opioides é abordada no Manual Diagnóstico e Estatístico de Transtornos Mentais em sua $5^{\mathfrak{a}}$ edição, o DMS$\mathrm{V}$, através dos Transtornos Relacionados a Opioides (APA, 2014).

\begin{abstract}
O transtorno por uso de opioides inclui sinais e sintomas que refletem a autoadministração compulsiva e prolongada de substâncias opioides usadas sem finalidade médica legítima, ou, na presença de outra condição médica cujo tratamento exige opioide, este é usado em doses muito acima da quantidade necessária (p. ex., um indivíduo com prescrição para opioides analgésicos com finalidade de alívio da dor em dosagem adequada fará uso significativamente maior do que indica a prescrição, e não apenas devido à dor persistente) (APA, 2014, p. 542).
\end{abstract}

O artigo intitulado "Retirada de Opioides: uma revisão bibliográfica" de Melo et al. (2020), publicado na revista Brazilian Journal of Development, teve por objetivo, através de revisão de literatura, revisar a farmacoterapia com a finalidade de descrever sobre os benefícios e os malefícios do uso de opioides bem como os efeitos de sua retirada. No estudo em questão, os autores versaram sobre a dependência e síndromes de abstinência severas como riscos inerentes ao uso contínuo de opioides e que trazem importantes implicações na saúde pública, alertando sobre a necessidade de mais pesquisas e estudos clínicos que possam pautar os profissionais prescritores deste tipo de medicação no seu uso seguro e adequado.

Segundo Calônego (2020), em sua pesquisa sobre as dificuldades sociais, legais e burocráticas para prescrição de opioides, da qual participaram 389 médicos, foi possível levantar que $52,19 \%$ dos profissionais possuem receio em prescrever este tipo de medicamento por medo de indução ao vício, $49,36 \%$ por medo de indução à dependência e 42,67\% afirmam ausência de conhecimento farmacológico sobre este princípio ativo. A partir de seu estudo, o autor concluiu que é essencial a reconstrução e a divulgação de conhecimento sobre os benefícios e sobre os riscos relacionados ao uso de opioides tanto para leigos quanto para os profissionais da saúde.

Bicca et al. (2012) estudaram sobre abuso e dependência dos opioides e opiáceos por meio de revisão bibliográfica, buscando auxiliar os profissionais médicos a reconhecerem e orientarem seus pacientes sobre o abuso dos opioides e opiáceos. Os autores apontaram que por se tratar de substâncias muito aditivas, podem apresentar 
alto potencial para a dependência e para a abstinência, principalmente diante de uma exposição e uso prolongado das mesmas. Adicionalmente, os autores alertam para o fato de que o uso crescente de medicamentos com estes princípios ativos configura-se como um problema de saúde pública que merece estudos e pesquisas contínuas que ofereçam conhecimento aos profissionais da área e à população como um todo.

Campos et al. (2020), através da publicação do artigo "Opioides: toxidade e efeitos indesejados" na Única Cadernos Acadêmicos, conduziram seu estudo através de pesquisa bibliográfica com o objetivo de fornecer informações a respeito do uso medicinal e/ou abusivo de opioides a profissionais de saúde e pacientes como foco nas suas consequências para o indivíduo, sinalizando que, embora sejam analgésicos eficientes, podem ocasionar fenômenos como tolerância, síndrome de abstinência e dependência. Assim como Melo et al. (2020), os pesquisadores alertaram para a relevância da realização de mais pesquisas sobre o tema por se tratar de um problema de saúde pública e também sobre a valia destas no sentido de orientar profissionais da saúde e pacientes sobre o uso de opioides de forma segura, orientada e racional.

Em estudo similar, Leal e Alencar (2020, p. 43) publicaram o artigo "Uso indevido e dependência de opioides: da prevenção ao tratamento" na Revista de Medicina de Família e Saúde Mental. A pesquisa, realizada através de revisão de literatura, teve por finalidade descrever sobre o uso indiscriminado e a dependência causada pelos opioides com base na preocupação crescente em relação ao uso destas substâncias. Para os autores, "em decorrência das prescrições excessivas dos opioides, o vício, overdoses e taxas de mortalidade atingiram proporções epidêmicas", se faz imprescindível a elaboração de mais pesquisas sobre a dor e sobre os riscos inerentes ao uso abusivo destas substâncias bem como oferecer reforço constante na educação dos profissionais prescritores das mesmas, buscando o "reconhecimento da dependência em tempo ágil, além, claro, da garantia de acesso aos tratamentos eficazes contra à adição”.

Vale ressaltar que, apesar dos achados teóricos revelarem a existência de diversos riscos relacionados ao uso indevido e/ou prolongado dos medicamentos opioides, o presente estudo teve como focos a dependência e a síndrome de abstinência como riscos potenciais neste contexto. 
Para fins de definição, importante conceituar uso indevido e uso prolongado. Este último, como o próprio nome evidencia, trata-se do uso por um longo período de tempo, independente do medicamento ter sido adequadamente prescrito ou não. Já o uso indevido é definido por Swift e Lewis (2010) como o uso inapropriado de medicamentos prescritos ou a sua utilização para finalidades que não sejam terapêuticas. Em concordância, a OMS (2020) o conceitua como o uso "não-médico", ou seja, aquele desacompanhado de prescrição, orientação e acompanhamento médico.

Entre as fontes pesquisadas para a presente pesquisa, foi possível constatar que dependência é citada como um risco relacionado ao uso de opioides por diversos autores: Soares et al. (2008), Swift e Lewis (2010), Nascimento e Sakata (2011), Sistema Único de Saúde de Santa Catarina (2015), Pereira, Andrade e Takitane (2016), Portela (2018), Melo et al. (2020), Leal e Alencar (2020) e Campos et al. (2020).

A dependência relacionada aos opioides também é abordada no DMS-V (APA, 2014) e na Classificação Estatística Internacional de Doenças e Problemas Relacionados com a Saúde, a CID-ıo (ONU, 1993).

Bicca et al. (2012, p. 7) apontam que os opioides agem como reforçadores positivos aplicados para cessar ou amenizar estados desagradáveis ou aversivos entendidos como reforços negativos, tais como a dor, a ansiedade e a depressão.

Nascimento e Sakata (2011), Pereira, Andrade e Takitane (2016), Leal e Alencar (2020) e Campos et al. (2020) confluem ao assinalar que o uso prolongado dos opioides ou sua administração contínua provoca alterações cerebrais estruturais e funcionais que podem trazer como resultado uma adaptação do organismo, fazendo com que o usuário se habitue a um novo estado de equilíbrio que depende da ação deste princípio ativo. Soares et al. (2008) caracterizam essa nova habituação do organismo aos opioides como um padrão mal adaptativo que pode provocar prejuízos ou sofrimento clinicamente significativos na vida do indivíduo.

Para Swift e Lewis (2010) e Nascimento e Sakata (2011), a dependência pode ser física e/ou psicológica. A dependência física pode ser compreendida justamente por essa habituação do organismo à presença dos opioides e reflete a necessidade deste princípio ativo na tentativa de manter o organismo em seu funcionamento já adaptado a este princípio ativo. 
Já a dependência psicológica é encarada como um fenômeno mais complexo, pois, de acordo com Swift e Lewis (2010), envolve o sistema de recompensa encefálico, ou seja, as sensações agradáveis que os opioides proporcionam ao indivíduo fazem com que este queria continuar usando a substância. Então, quando o seu uso é cessado ou minimizado, o sistema de recompensa encefálico já habituado às sensações agradáveis, se altera apresentando, por exemplo, sinais de ansiedade, agitação e fissura, ou seja, uma vontade muito forte ou compulsiva pelas sensações provocadas pelo uso dos opioides.

Melo et al. (2020) destacam que o grande desafio científico em relação ao uso dos opioides está na prescrição deste princípio ativo para que este atinja sua finalidade terapêutica, ou seja, o tratamento de dores agudas e crônicas, buscando reduzir ao máximo riscos como a tolerância a dependência.

A síndrome da abstinência, por sua vez e assim como a dependência, é elencada como um risco associado ao relacionado ao uso de opioides por diversos autores: Soares et al. (2008), Swift e Lewis (2010), Nascimento e Sakata (2011), Bicca et al. (2012), SUS/SC (2015), Pereira, Andrade e Takitane (2016), O’Malley e O’Malley (2020) e

Campos et al. (2020).

A referida síndrome pode ser caracterizada pela manifestação de diversos sintomas, físicos e/ou psicológicos, quando há cessação ou redução brusca no uso pesado ou prolongado de opioides (NASCIMENTO; SAKATA, 20II; APA, 2014).

A abstinência de opioides está presente no DMS-V, sendo classificada dentro dos Transtornos Relacionados a Opioides - Abstinência de Opioides. O manual traz em seu conteúdo que tal síndrome se caracteriza por um padrão de sinais e sintomas e apresenta os critérios diagnósticos para este transtorno (APA, 2014).

Soares et al. (2008), SUS/SC (2015) e Campos et al. (2020) apontam entre os sintomas da síndrome de abstinência: desejo do fármaco, elevação da pressão arterial, taquicardia, ansiedade e humor deprimido, irritabilidade, sudorese, bocejo, lacrimejamento, febre, aumento da intensidade da dor, dores musculares, náusea, vômito, cólicas abdominais, diarreia, tremor, piloereção, perda de energia, insônia ou sono agitado, espasmos musculares, anorexia, calafrios, entre outros. Acrescenta o 
SUS/SC (2015) que os sintomas da abstinência são extremamente desconfortáveis e podem causar sofrimento no indivíduo que os sente.

Pereira, Andrade e Takitane (2016) alertam para o fato de que os sintomas da abstinência podem levar o indivíduo a ingerir doses inadequadas de opioides no desespero de cessar os sintomas e que, portanto, tal situação merece atenção especial, pois pode levar a uma possível overdose.

\section{CONCLUSÃO}

Os opioides são medicamentos de escolha para dores agudas e crônicas por possuírem alto poder analgésico e hipnótico. Por outro lado, o seu uso prolongado e/ou inadequado pode oferecer riscos diversos ao seu usuário.

A partir da presente pesquisa, foi possível constatar que, entre os riscos oferecidos pelo uso indevido de opioides, as evidências teóricas apontam a dependência e a síndrome de abstinência, entre outros, tais como a tolerância, o vício, a overdose e até mesmo o óbito.

Ainda, ficou claro que atualmente existe uma grande preocupação em relação

ao uso de opioides tendo em vista o dualismo que este uso apresenta. Por um lado, a sua indicação auxilia com a diminuição dos quadros de dores consideradas agudas e crônicas, oferecendo benefícios aos seus usuários. Por outro lado, o uso incorreto acarreta em efeitos adversos.

Diante do exposto, cabe a reflexão da necessidade de mais pesquisas sobre o fenômeno da dor e sobre o uso dos opioides bem como o reforço constante na educação de quem os prescreve, buscando uma orientação adequada e segura em relação a sua administração e posologia e capacitando-os no sentido de detectar, de forma breve, a presença de dependência, síndrome de abstinência, entre outras consequências, garantindo a segurança do tratamento proposto.

\section{REFERÊNCIAS}

AMERICAN PSYCHIATRIC ASSOCIATION (APA). Manual diagnóstico e estatístico de transtornos mentais: DSM-5. Tradução de Maria Inês Corrêa Nascimento et al. 5. ed. Porto Alegre: Artmed, 2014. 
ANTUNES, Felipe. Medicamentos opioides - o que preciso saber? Cartas ao Editor, Acta Med Port, Portugal, v. 31, n. 12, p. 796-798, dez. 2018.

BARROS, Guilherme Antônio Moreira de et al. Uso de analgésicos e o risco da automedicação em amostra de população urbana: estudo transversal. Rev. Bras. Anestesiol., Campinas, v. 69, n. 6, p. 529-536, dez. 2019. ISSN o034-7094.

BICCA, Carla Hervê Moram et al. Abuso e Dependência dos Opioides e Opiáceos. Projeto Diretrizes. Associação Brasileira de Psiquiatria, Sociedade Brasileira de Patologia Clínica e Medicina Laboratorial, Sociedade Brasileira de Medicina de Família e Comunidade. São Paulo: Associação Médica Brasileira, 2012. Disponível em: 〈https://diretrizes.amb.org.br/_BibliotecaAntiga/abuso_e_dependencia_de_opioides. pdf $>$. Acesso em io mar. 202I.

CALÔNEGO, Marco A. Marchetti. Dificuldades sociais, legais e burocráticas para

prescrição de opioides. Tese (Doutorado em Anestesiologia) - Faculdade de Medicina, Universidade Estadual Paulista Júlio de Mesquita Filho. Botucatu, SP, p. 93, 2020.

CAMPOS, Helaine Sinezia Pinto et al. Opioides: toxidade e efeitos indesejados. Única Cadernos Acadêmicos, Ipatinga, v. 3, n. I, ano 6, jun./set. 2020. ISSN 2594-9624.

CONSELHO FEDERAL DE FARMÁCIA (CFF). Uso prolongado de analgésicos traz riscos à saúde. Brasília, jun. 2016. Disponível em: 〈https://www.cff.org.br/noticia.php?id=3827\&titulo=Uso+prolongado + de + analg\%C 3\%A9sicos+traz+riscos+\% $3 \%$ Ao+sa\% $\mathrm{C}_{3} \% \mathrm{BAde}>$. Acesso em io mar. 2021.

KRAYCHETE, Durval Campos; GARCIA, João Batista Santos; SIQUEIRA, José Tadeu Tesseroli de. Recomendações para uso de opioides no Brasil: Parte IV. Efeitos adversos de opioides. Rev. dor, São Paulo, v. 15, n. 3, p. 215-223, set. 2014. 
LEAL, Raphael S.; ALENCAR, Guilherme A. de B. C. Uso indevido e dependência de opioides: da prevenção ao tratamento. Revista de Medicina de Família e Saúde Mental - UNIFESO, Rio de Janeiro, v. 2, n. I, p. 29-44, 2020. ISSN 2674-7219.

MELO, Andressa Piva de et al. Retirada de opioides: uma revisão bibliográfica. Braz. J. of Develop., Curitiba, v. 6, n. 9, p. 67098-67112, set. 2020.

NASCIMENTO, Daiana Ciléa Honorato; SAKATA, Rioko Kimiko. Dependência de opioide em pacientes com dor crônica. Rev Dor., São Paulo, v. I2, n. 2, p. 160-165, abr./jun. 20II.

PEREIRA, Mariana de Moura; ANDRADE, Letycia de Paiva; TAKITANE, Juliana. Evolução do uso abusivo de derivados de ópio. Saúde, Ética \& Justiça, São Paulo, v. 2I, n. I, p. 12-17, 2016. ISSN 2317-2770.

PORTELA, Graça. Uso de opiáceos para combater dores crônicas cresce $465 \%$.

Fundação Oswaldo Cruz (FIOCRUZ), jun. 2018. Disponível em: <https://portal.fiocruz.br/noticia/uso-de-opiaceos-para-combater-dores-cronicascresce-465>. Acesso em o6 mar. 2021.

O’MALLEY, Gerald. F.; O’MALLEY, Rika. Opioides. Assuntos especiais - Manual MSD Versão Saúde para a Família, jun. 2020. Disponível em: 〈https://www.msdmanuals.com/pt/casa/assuntos-especiais/drogas-recreativas-eentorpecentes/opioides $>$. Acesso em 15 mar. 2021.

ORGANIZAÇÃO MUNDIAL DE SAÚDE (OMS). Classificação de Transtornos Mentais e de Comportamento da CID-ıo: descrições clínicas e diretrizes diagnósticas. Tradução de Dorgival Caetano. Porto Alegre: Artmed, 1993. 
ORGANIZAÇÃO MUNDIAL DE SAÚDE (OMS). Overdose de opioide. 28 de agosto de 2020. Disponível em: <https://www.who.int/news-room/factsheets/detail/opioid-overdose> Acesso em: 06 mar. 2021.

SECRETARIA DE ATENÇÃO À SAÚdE. MINISTÉRIO DA SAÚdE. PORTARIA № r.o83, DE 2 DE OUTUBRO DE 2012. Aprova o Protocolo Clínico e Diretrizes Terapêuticas da Dor Crônica. Diário Oficial [da] República Federativa do Brasil, Brasília, DF, 03 out. 2012. № I083, Seção I, p. 54 .

SECRETARIA DE VIGILÂNCIA EM SAÚDE. MINISTÉRIO DA SAÚDE. PORTARIA № 344, DE 12 DE MAIO DE 1998. Aprova o Regulamento Técnico sobre substâncias e medicamentos sujeitos a controle especial. Diário Oficial [da] República Federativa do Brasil, Brasília, DF, I9 mai. 1998. Seção I, p. 6r.

SISTEMA ÚNICO DE SAÚdE (SUS/SC). Estado de Santa Catarina. Abuso e dependência de derivados do ópio: protocolo clínico. Santa Catarina, 2015. Disponível

em: 〈https://www.saude.sc.gov.br/index.php/documentos/atencao-basica/saudemental/protocolos-da-raps/9213-dependencia-de-derivados-do-opio/file>. Acesso em Io mar. 2021.

SOARES, Hugo Leonardo Rodrigues et al. Dependência de opioides: uma revisão da literatura. Monografia (Especialização em Dependência Química) - Universidade Federal de São Paulo, SP, p. 37, 2008.

SOUZA, Juliana Barcellos de; BARROS, Carlos Marcelo de. Considerações sobre o novo conceito de dor. BrJP, São Paulo, v. 3, n. 3, p. 294, set. 2020. ISSN 2595-orr8.

SWIFT, Robert M.; LEWIS, David C. Farmacologia da dependência e abuso de drogas. In: GOLAN, David E. et al. Princípios de farmacologia: a base fisiopatológica da farmacoterapia. 2 ed. Rio de Janeiro: Guanabara Koogan, 2010, Cap. 17, p 260-278. 